discontinuation was 7 (range: 2-56) and 10 (range: 2-48), respectively. Pre-infusion steroids were re-started in 3 patients after a rise in SUA, with 1 regaining sUA response (patient 2) and 2 discontinuing therapy (patients 4 and 9). An additional patient had a loss of sUA response (patient 7) and discontinued, 1 patient discontinued due to hospitalization (patient 6), and 1 patient chose to discontinue therapy (patient 8). At the time of data collection, 4 patients remained on therapy (patients 1-3,5). Eight patients had at least one AE (all deemed unrelated to treatment), including influenza, hypertensive crisis, and toe amputation. No IRs occurred

Conclusion: In this case series, 8 of 9 patients received 6 months or more $(\geq 12$ infusion) of pegloticase. These cases suggest that pre-infusion GC discontinuation may be possible in some patients treated with pegloticase (particularly those over 70 years). Further investigation exploring this concept is warranted, including evaluating the optimal time and conditions to discontinue pre-infusion GCs with pegloticase.

REFERENCES:

[1] Sundy JS, et al. JAMA. 2011;306(7):711-720.

[2] KRYSTEXXA (pegloticase) [prescribing information] Horizon.

[3] Saag KG. Bull NYU Hosp Jt Dis 2012;70(Suppl 1):S21-S25.

[4] Pillinger MH, et al. Bull NYU Hosp Jt Dis 2010;68:199-203.

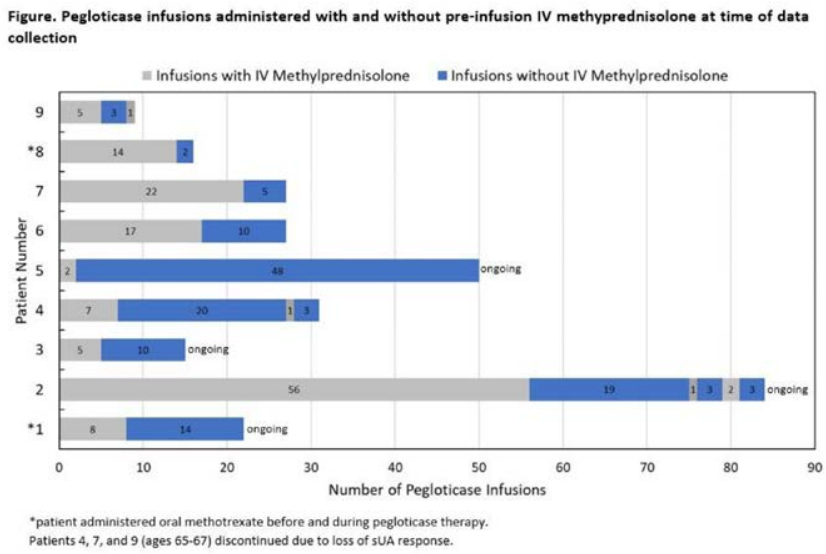

Disclosure of Interests: Veronica Newsome Speakers bureau: Abbvie, Anthony Amatucci Shareholder of: Horizon Therapeutics plc, Employee of: Horizon Therapeutics plc, Tim Stainbrook: None declared., Brian LaMoreaux Shareholder of: Horizon Therapeutics plc, Employee of: Horizon Therapeutics plc.

DOI: 10.1136/annrheumdis-2021-eular.70

\section{AB0629 1 ACCURACY OF SYNOVIAL FLUID ANALYSIS FOR THE IDENTIFICATION OF CALCIUM PYROPHOSPHATE CRYSTALS: AN ANCILLARY STUDY OF OMERACT CRITERION VALIDITY STUDY FOR ULTRASOUND IN CPPD}

A. Adinolfi ${ }^{1}$, S. Sirotti ${ }^{2}$, M. Gutierrez ${ }^{3}$, C. Pineda ${ }^{3}$, D. Clavijo Cornejo ${ }^{3}$, T. Serban ${ }^{4}$, A. Dumitru', A. Scanu', M. A. D'agostino ${ }^{7}$, H. Keen ${ }^{8}$, L. Terslev ${ }^{9}$, P. Sarzi-Puttini ${ }^{2}$, C. A. Scirè ${ }^{10}$, A. Iagnocco ${ }^{11}$, G. Filippou ${ }^{2}$ on behalf of Omeract Ultrasound working group, subgroup CPPD.

${ }^{1}$ ASST Grande Ospedale Metropolitano Niguarda, Rheumatology Unit, Milan, Italy; ${ }^{2}$ Luigi Sacco University Hospital, Rheumatology Department, Milan, Italy; ${ }^{3}$ Instituto Nacional de Rehabilitacion, Division of Musculoskeletal and Rheumatic Diseases, Mexico City, Mexico; ${ }^{4}$ Ospedale La Colletta, ASL3 Genovese, Rheumatology Department, Genoa, Italy; ${ }^{5}$ Carol Davila University of Medicine and Pharmacy, Department of Pathology, Bucharest $r$, Romania; ${ }^{6}$ University of Padova, Department of Medicine-DIMED, Rheumatology Unit, Padova, Italy; ${ }^{7}$ Università Cattolica del Sacro Cuore, Policlinico Universitario Agostino Gemelli IRCSS, Rheumatology Department, Rome, Italy; ${ }^{8}$ The University of Western Australia, Medicine and Pharmacology Department, Murdoch, Perth, Australia; ${ }^{9}$ Copenhagen University, Center for Rheumatology and Spine Diseases, Rigshospitalet, Copenhagen, Denmark; ${ }^{10}$ Società Italiana di Reumatologia, Epidemiology Research Unit, Milan, Italy; ${ }^{11}$ Università degli Studi di Torino,
Rheumatology Unit, Department of Clinical and Biological Sciences, Torino, Italy

Background: Synovial fluid analysis (SFA) via compensated polarized light microscopy is still considered the gold standard for the identification and diagnosis of Calcium Pyrophosphate Deposition disease (CPPD)-related arthropathies[1], but very few studies have been published about its diagnostic accuracy.

Objectives: The aim of this study was to evaluate the accuracy of SFA in the identification of calcium pyrophosphate dihydrate (CPP) crystals compared to microscopic analysis of joint tissues as the reference standard.

Methods: This is an ancillary study of an international, multicentre cross-sectional study performed by the CPPD subgroup of the OMERACT Ultrasound working group[2]. Consecutive patients with knee osteoarthritis (OA) waiting for total knee replacement surgery were enrolled in the study from 2 participating centres, Mexico and Romania. During surgical procedures synovia fluid (SF), menisci and hyaline cartilage were collected and analysed within 48 hours after surgery under transmitted light microscopy and compensated polarised light microscopy for the presence/absence of CPP crystals. All slides were analysed by expert examiners on site, blinded to other findings. A dichotomic score (absence/presence) was used for scoring both SF and tissues. Microscopic analysis of knee tissues was considered the gold standard. Sensitivity, specificity, accuracy, positive and negative predictive values (PPV and NPV) of SFA in the identification of CPP crystals were calculated.

Results: 15 patients $(53 \%$ female, mean age $68 y 0 \pm 8.4)$ with OA of grade 3 or 4 according to Kellgren-Lawrence scoring were enrolled. 12 patients $(80 \%)$ were positive for CPP crystals at SFA and 14 (93\%) at tissues microscopic analysis. Among 12 SFA positive patients, all were positive for CPP crystals in either medial or lateral meniscus, and 11 were positive in both; 10 patients were positive at the hyaline cartilage, and all 10 were also positive for at least one meniscus Regarding the 3 SFA negative patients, only one had no crystals in the examined tissues, while the other 2 patients had CPP crystals in both menisci and hyaline cartilage. The overall diagnostic accuracy of SFA compared to histology analysis for CPPD was $87 \%$, with a sensitivity of $86 \%$ and a specificity of $100 \%$, the PPV was $100 \%$ and the NPV was $33 \%$ (Table 1).

Table 1. sensitivity, specificity, positive predictive value (PPV), negative predictive value (NPV) and diagnostic accuracy of synovial fluid analysis compared to the reference standard. Cl: Confidential Interval. SF: synovial fluid, in parentheses: numerators and denominators for all percentages provided.

\begin{tabular}{cccccc}
\hline & Sensitivity & Specificity & PPV & NPV & Accuracy \\
\hline SF analysis & $86 \%(12 / 14)$ & $100 \%(1 / 1)$ & $100 \%(12 / 12)$ & $33 \%(1 / 3)$ & $87 \%(13 / 15)$ \\
& $(0.65-0.99) \mathrm{Cl}$ & $(0.0-0.25) \mathrm{Cl}$ & $(0.65-0.99) \mathrm{Cl}$ & $(0.0-0.25) \mathrm{Cl}$ & \\
$95 \%$ & $95 \%$ & $95 \%$ & $95 \%$ & \\
\hline
\end{tabular}

Conclusion: SFA demonstrated to be an accurate test for the identification of CPP crystals in patients with advanced OA. However, is not always feasible and carries some risks for the patient. Considering the availability of validated imaging techniques for the detection of CPPD, such as US, SFA could be used in those patients where imaging and clinical data are not definitely confirmatory of the disease.

REFERENCES:

[1] W. Zhang et al., 'European League Against Rheumatism recommendations for calcium pyrophosphate deposition. Part I: terminology and diagnosis', Ann Rheum Dis, vol. 70, no. 4, pp. 563-570, Apr. 2011, doi: 10.1136/ ard.2010.139105

[2] G. Filippou et al., 'Criterion validity of ultrasound in the identification of calcium pyrophosphate crystal deposits at the knee: an OMERACT ultrasound study', Ann Rheum Dis, p. annrheumdis-2020-217998, Sep. 2020, doi: 10.1136/annrheumdis-2020-217998.

Disclosure of Interests: None declared.

DOI: 10.1136/annrheumdis-2021-eular.601

\begin{tabular}{|l|l|}
\hline AB0630 & PEGLOTICASE/METHOTREXATE CO-THERAPY \\
IMPROVED JOINT AND PATIENT-REPORTED HEALTH \\
ASSESSMENTS IN PATIENTS WITH UNCONTROLLED \\
GOUT: 12-MONTH EXPLORATORY OUTCOMES OF THE \\
MIRROR OPEN-LABEL TRIAL
\end{tabular}

J. Botson ${ }^{1}$, P. M. Peloso ${ }^{2}$, K. Obermeyer ${ }^{2}$, B. Lamoreaux ${ }^{3}$, L. Zhao' ${ }^{2}$ M. E. Weinblatt ${ }^{4}$, J. Peterson ${ }^{5}{ }^{1}$ Orthopedic Physicians Alaska, Medical, 
Anchorage, United States of America; ${ }^{2}$ Horizon Therapeutics plc, Research and Development, Deerfield, United States of America; ${ }^{3}$ Horizon Therapeutics plc, Medical Affairs, Deerfield, United States of America; ${ }^{4}$ Brigham and Women's Hospital, Division of Rheumatology, Immunology and Allergy, Boston, United States of America; ${ }^{5}$ Western Washington Medical Group, Arthritis Clinic, Bothell, United States of America

Background: Gout development follows persistent serum uric acid (sUA) elevation. Patients who are refractory to or cannot tolerate oral urate lowering therapies (ULTs) have limited treatment options. Pegloticase is effective in treating refractory gout, but many patients develop anti-drug antibodies (ADAs), which are associated with loss of urate-lowering efficacy ${ }^{1-3}$ and infusion reactions (IRs). ${ }^{1,2}$ In phase 3 trials, the pooled pegloticase responder rate during Months 3 and 6 combined was $42 \%$ ( $8 \mathrm{mg}$ infusion every 2 weeks), with high-titer ADA positive patients losing efficacy prior to 6 months. ${ }^{1}$ The 6 -month results from the MIRROR open-label trial (79\% response rate [11/14], 95\% Cl 49-95\%) ${ }^{4}$ suggest that methotrexate (MTX) administered in conjunction with pegloticase increases treatment responder rate.

Objectives: To examine longer-term (12-month) exploratory endpoints from the MIRROR open-label trial, including joint, overall health, and gout global assessments. Serial dual-energy computed tomography (DECT) images were also examined when available.

Methods: Adult patients with uncontrolled gout (sUA $\geq 6 \mathrm{mg} / \mathrm{dL}$ with $\geq 1$ of the following: sUA $\geq 6 \mathrm{mg} / \mathrm{dL}$ despite ULT use, intolerance to ULT, or functionally limiting tophaceous deposits) were included. Patients with immunocompromised status, G6PD deficiency, severe renal impairment, or MTX contraindication were excluded. Patients were administered oral MTX (15 mg/week) and folic acid ( $1 \mathrm{mg} /$ day) 4 weeks prior to and throughout pegloticase therapy ( $8 \mathrm{mg}$ biweekly infusion for up to 52 weeks). Exploratory outcomes included mean change from baseline (CFB) in number of affected joints (tophi, swollen, tender), Health Assessment Questionnaire (HAQ) scores (Disability Index [DI; score 0-3], Pain [score 0-100], Health [score 0-100]), and Gout Global Assessments (Patient, Physician; score 0-10). A decrease in these measures reflects clinical/ patient-reported health improvement. Change in urate deposition volume, as measured on DECT imaging, was also examined as available. Analyses were performed on the modified intent-to-treat ( $\mathrm{mlTT}$ ) population ( $\geq 1$ pegloticase infusion received)

Results: 14 patients (all male, mean $\pm S D$ age: $49.3 \pm 8.7$ years) made up the $\mathrm{mITT}$ population. Mean $\pm \mathrm{SD}$ sUA prior to pegloticase treatment was $9.2 \pm 2.5 \mathrm{mg} / \mathrm{dL}$ and 13 patients had visible tophi. 3 patients discontinued due to 2 consecutive sUA levels $>6 \mathrm{mg} / \mathrm{dL}$ and 1 patient completed the study at week 24 (pre-protocol amendment extending treatment from 24 to 52 weeks). 10 patients completed the 52-week study. Of these, 8 patients received 26 infusions and 2 patients received 12 infusions, discontinued pegloticase after meeting their treatment goal at 24 weeks, and started allopurinol while remaining in study under observation. At week $52(n=10$, sUA $=1.1 \pm 2.5 \mathrm{mg} /$ $\mathrm{dL}$ ), the number of affected joints improved, along with $\mathrm{HAQ}$ measures (Figure 1). Global Assessments of Gout also improved (Physician: CFB=5.7 \pm 2.6 , Patient $C F B=-4.6 \pm 2.1$ ) and majority of subjects had a score of 0 or 1 ( $0=$ "excellent health") at week 52 (Physician: $0.3 \pm 0.5$, Patient: $1.1 \pm 1.3$ ). Two patients had available DECT imaging. One received pegloticase/methotrexate co-therapy thru week 52 and had a marked reduction in total urate volume (baseline: $128.76 \mathrm{~cm}^{3}$, week $52: 1.33 \mathrm{~cm}^{3}$ ). The other received only 5 pegloticase infusions, but also showed total urate volume reduction (baseline: $59.20 \mathrm{~cm}^{3}$, week 10: $25.07 \mathrm{~cm}^{3}$ ). Both patients displayed improvement in bone erosion healing.

Conclusion: These 12-month exploratory endpoints of the MIRROR open-label trial suggest that MTX/pegloticase co-therapy results in meaningful changes in clinical evaluations (tophaceous, tender, and swollen joint counts), and patient-reported outcomes (pain, disability) in patients with uncontrolled gout.

\section{REFERENCES}

[1] Sundy JS et al. JAMA 2011;306:711-20

[2] Baraf HS et al. J Clin Rheumatol 2014;20:427-32
[3] Lipsky PE et al. Arthritis Res Ther 2014, 16:R60

[4] Botson JK et al. J Rheum 2020 [Epub ahead of print]
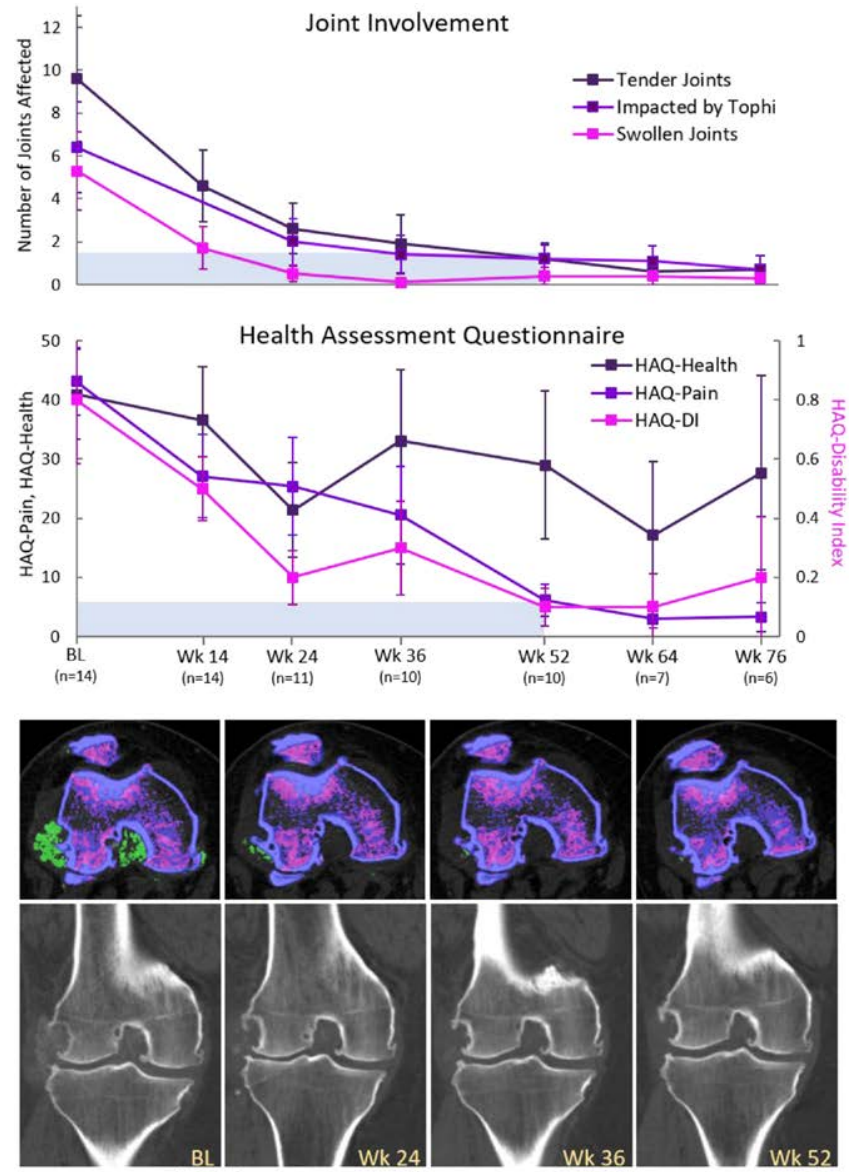

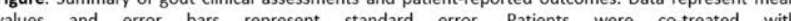

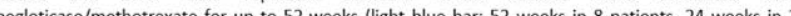
patients). Week 64 and 76 represent the 3 and 6 month follow-up visits, respectively. DECT imaging shows progressive urate volume decrease in this knee (BL: $22.6 \mathrm{~cm}^{3}$, Week 52: $0.1 \mathrm{~cm}^{3}$ ) and bone erosion healing concomitant with clinical improvement. BL, baseline; $\mathrm{HAQ}$ health assessment questionnaire; $\mathrm{DI}$, disability index

Disclosure of Interests: John Botson Speakers bureau: Horizon Therapeutics, Celgene, Novartis, and AbbVie, Consultant of: Horizon Therapeutics, Celgene, Novartis, and AbbVie, Grant/research support from: Horizon Therapeutics and Radius Health, Paul M. Peloso Shareholder of: Horizon Therapeutics plc, Employee of: Horizon Therapeutics plc, Katie Obermeyer Shareholder of: Horizon Therapeutics plc, Employee of: Horizon Therapeutics plc, Brian LaMoreaux Shareholder of: Horizon Therapeutics plc, Employee of: Horizon Therapeutics plc, Lin Zhao Shareholder of: Horizon Therapeutics plc, Employee of: Horizon Therapeutics plc, Michael E. Weinblatt Shareholder of: Lycera, Can-Fite BioPharma, Scipher Medicine, Inmedix, and Vorso, Consultant of: Bristol Myers Squibb, Corona, Lilly, AbbVie, Amgen, Arena, GlaxoSmithKline, Gilead Sciences, Horizon Therapeutics, Lycera, Novartis, Pfizer, Roche, Samsung, Scipher Medicine, and Set Point, Grant/research support from: Amgen, Bristol-Myers Squibb, Crescendo Bioscience, Lilly and Sanofi, Jeff Peterson Speakers bureau: Horizon Therapeutics plc, Grant/research support from: Horizon Therapeutics plc. DOI: 10.1136/annrheumdis-2021-eular.996 